\title{
Investigation of the vertical instability at the Argonne Intense Pulsed Neutron Source
}

\author{
Shaoheng Wang* \\ Institute of Fluid Physics, China Academy of Engineering Physics, Mianyang, 621900, China \\ J. C. Dooling, K. C. Harkay, R. L. Kustom, and G. E. McMichael \\ Argonne National Laboratory, 9700 South Cass Avenue, Argonne, Illinois 60439, USA \\ (Received 23 March 2009; published 27 October 2009)
}

\begin{abstract}
The rapid cycling synchrotron of the intense pulsed neutron source at Argonne National Laboratory normally operates at an average beam current of 14 to $15 \mu \mathrm{A}$, accelerating protons from 50 to $450 \mathrm{MeV}$ 30 times per second. The beam current is limited by a single-bunch vertical instability that occurs in the later part of the $14 \mathrm{~ms}$ acceleration cycle. By analyzing turn-by-turn beam position monitor data, two cases of vertical beam centroid oscillations were discovered. The oscillations start from the tail of the bunch, build up, and develop toward the head of the bunch. The development stops near the bunch center and oscillations remain localized in the tail for a relatively long time (2-4 ms, $1-2 \times 10^{4}$ turns). This vertical instability is identified as the cause of the beam loss. We compared this instability with a head-tail instability that was purposely induced by switching off sextupole magnets. It appears that the observed vertical instability is different from the classical head-tail instability.
\end{abstract}

DOI: 10.1103/PhysRevSTAB.12.102802

PACS numbers: 29.27.Bd, 29.20.dk, 41.75.Ak, 41.85.Qg

\section{INTRODUCTION}

The intense pulsed neutron source (IPNS) accelerator system at Argonne National Laboratory consists of an $\mathrm{H}^{-}$ ion source, a Cockcroft-Walton preaccelerator, a $50 \mathrm{MeV}$ Alvarez linac, a rapid cycling synchrotron (RCS), transport lines, and ancillary subsystems (controls, diagnostics). The accelerator normally operates at an average beam current of 14 to $15 \mu \mathrm{A}$, delivering pulses of approximately $3.0 \times$ $10^{12}$ protons at $450 \mathrm{MeV}$ to the target, 30 times per second. Time in the RCS acceleration cycle is referenced to $B_{\min }$, the minimum of the magnetic guide field $(t=0)$. A $70 \mu \mathrm{s}$ macropulse is used to fill the RCS in coasting beam mode starting $300 \mu$ s prior to $B_{\min }$. During injection, the $\mathrm{H}^{-}$ions are stripped to protons in a $55 \mu \mathrm{g} / \mathrm{cm}^{2}$ carbon foil.

The RCS is a ring-type accelerator with sixfold symmetry and combined-function magnets. Two quadrupoles, one for each transverse plane, and two pairs of sextupoles are employed for tune and chromaticity control. Two rf cavities accelerate a single proton bunch $(h=1)$ from 50 to $450 \mathrm{MeV}$ while the rf frequency varies from 2.21 to $5.14 \mathrm{MHz}$ in $14.2 \mathrm{~ms}$. Throughout the acceleration period, the RCS operates below its transition energy. Some design parameters of the IPNS RCS are shown in Table I.

At an injected charge of $3.6-3.7 \times 10^{12}$ protons $(0.58-0.59 \mu \mathrm{C})$, a vertical instability starts to grow approximately $10 \mathrm{~ms}$ after injection. The oscillation, if left unchecked, causes serious beam loss. Although this beam loss mechanism has existed for many years, the detailed

\footnotetext{
*wangshaoheng@yahoo.com
}

physical process of the instability has never been fully understood. In the operation of the RCS, the instability is suppressed by phase modulation (PM) of the rf voltage. The PM increases the energy spread of the proton beam and, hence, suppresses the instability via Landau damping. PM leads to a strong breathing mode oscillation (10$11 \mathrm{kHz}$ ) in the longitudinal profile of the beam.

Earlier studies employed frequency domain analysis. The results clearly showed the existence of the instability. In recent studies, turn-by-turn beam position monitor (BPM) data were extracted from the raw data and showed the development of the instability. We found that the vertical oscillations of the bunch centroid start at the tail and are localized in this region for the remaining $4 \mathrm{~ms}$ until extraction. This phenomenon is quite different from what is observed during a classical head-tail instability [1], which had been considered as one of the possible mechanisms of the instability at the RCS.

TABLE I. IPNS RCS parameters.

\begin{tabular}{lc}
\hline \hline Parameters & Values \\
\hline Circumference & $42.95 \mathrm{~m}$ \\
Injection energy & $50 \mathrm{MeV}$ \\
Extraction energy & $450 \mathrm{MeV}$ \\
Harmonic number & 1 \\
Number of periods & 6 \\
Tune $x$ & 2.2 \\
Tune $y$ & 2.32 \\
Maximum rf voltage & $21 \mathrm{kV}$ \\
Transition $\gamma$ & 2 \\
\hline \hline
\end{tabular}



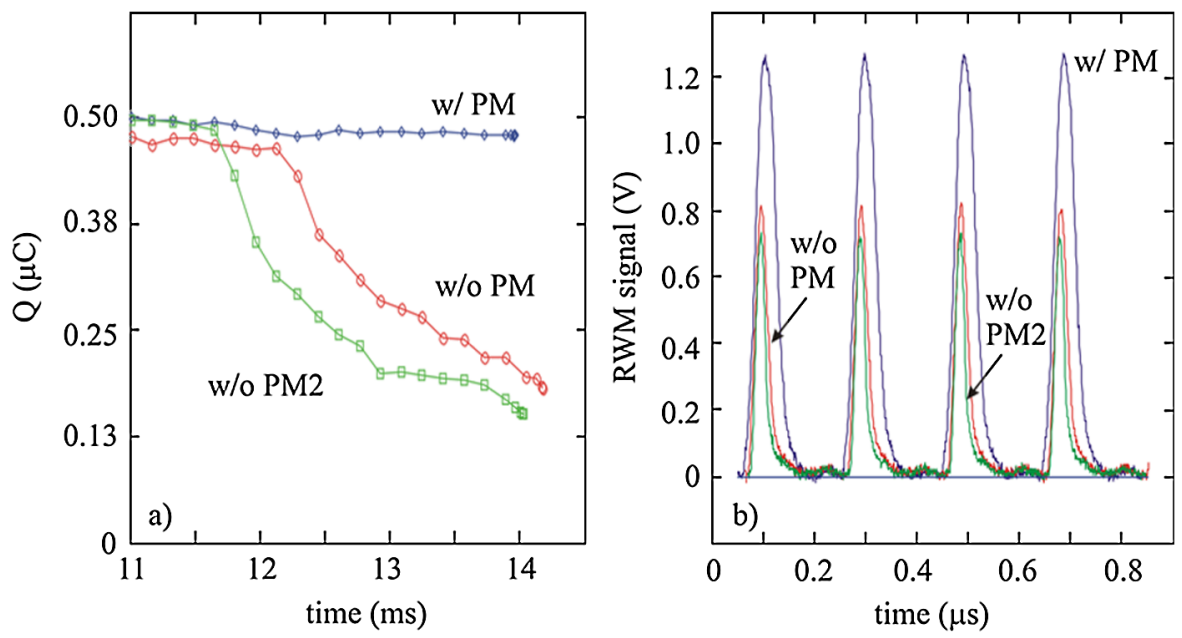

FIG. 1. (Color) RWM data: (a) Integrated current showing charge behavior with rf phase modulation and for two cases without phase modulation. Note that beam loss begins earlier for the higher charge cycle; (b) instantaneous current signals for the same three cases shown in (a) shortly before extraction.

\section{BEAM LOSS AND RF PM}

As mentioned in the previous section, the phenomenon of vertical instability and beam loss have been noticed since the early days of operating the RCS [2]. The vertical sidebands during the instability occur at $\frac{|\Delta f|}{f}=0.25$. During more recent studies, it was also found to be difficult to prevent the vertical tune from crossing this value late in the cycle.

Data from a resistive wall monitor (RWM) diagnostic are shown in Fig. 1, for acceleration cycles with and without the instability. The integrated RWM waveform in Fig. 1(a) is proportional to the bunch charge. When rf PM is off, significant particle loss begins about $12 \mathrm{~ms}$ after injection and the bunch is shortened longitudinally. Note that beam loss begins earlier in the acceleration cycle with higher charge. RWM waveforms just prior to extraction are presented in Fig. 1(b).

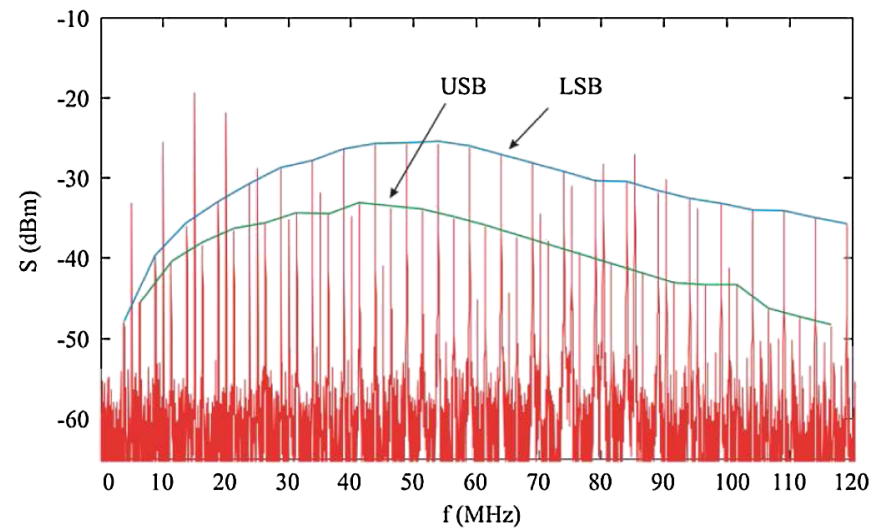

FIG. 2. (Color) FFT results when beam loss occurs, the width of time window is $37.5 \mu \mathrm{s}$, data were taken at $12.88 \mathrm{~ms}$ after injection. USB: upper sideband. LSB: lower sideband.
First, we did studies in the frequency domain. Since the proton bunch revolution frequency increases from $2.21 \mathrm{MHz}$ at injection to $5.14 \mathrm{MHz}$ at extraction in $14 \mathrm{~ms}$, the sampling window of BPM data for fast Fourier transform (FFT) cannot be too wide; otherwise, the shifting of the revolution frequency will blur the FFT results. We limited the time window width to be less than $50 \mu$ s to avoid this effect. Spectra are obtained from FFTs of time data recorded on a deep-memory oscilloscope sampling at a rate of $1.25 \times 10^{9}$ samples per second. When beam loss occurs, we can see in the FFT that the amplitudes of the lower sidebands are much stronger than those of the upper sidebands, as shown in Fig. 2. This indicates that a slow-wave instability is developing [3]. In fact, this character of the spectrum also appears before the beam loss happens, which means that the instability comes before the beam loss. Figure 3 shows the moment in the cycle when the BPM data time window was taken for the FFT results presented in Fig. 2. The amplitude of

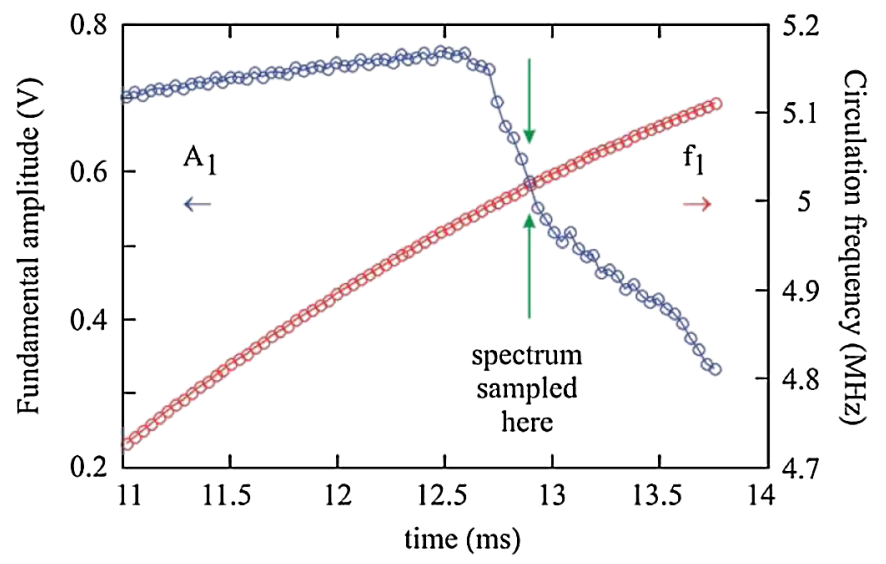

FIG. 3. (Color) The moment when the BPM data time window was taken for the FFT process. 

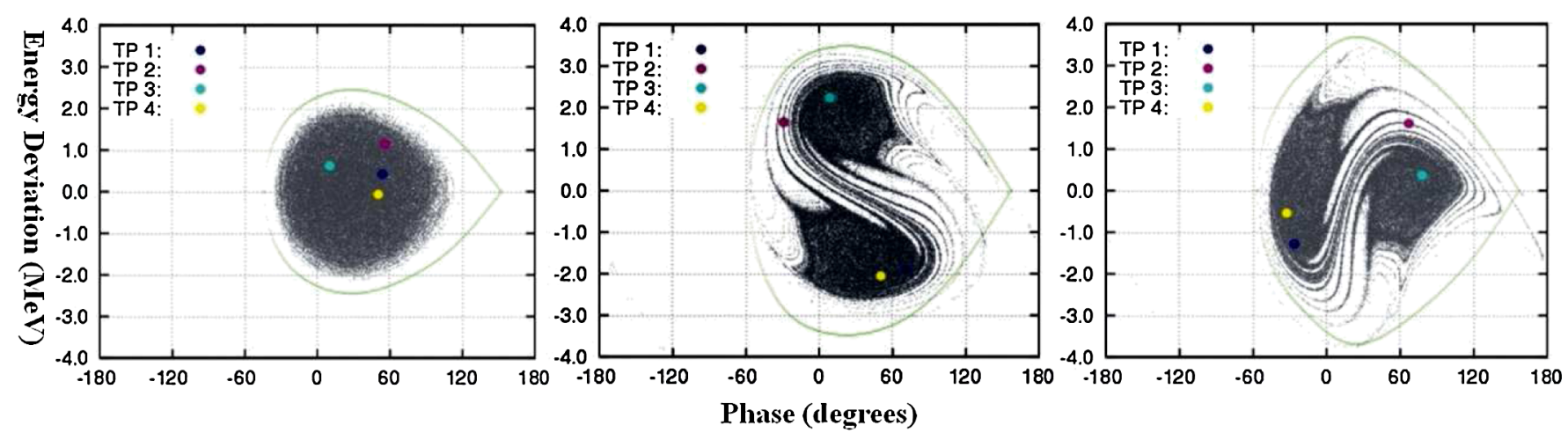

FIG. 4. (Color) Simulation results of dynamics in longitudinal phase space. The left graph is when rf PM is off. The center and right graphs, rf PM is on, two typical particle distributions are shown for different moments in the synchrotron motion. TP indicates test particle.

fundamental component is shown to indicate that the data were taken when beam loss occurred. The revolution frequency at that moment is about $5 \mathrm{MHz}$, not long before extraction.

In daily operations, the instability is suppressed by PM of the two rf cavities [4]. The rf PM introduces an additional disturbance term in the Hamiltonian of the motion in longitudinal phase space [5]. The driving terms of nonlinear resonances drive only odd order parametric resonances. The purpose of rf PM is to increase the momentum spread of the bunch and thereby provide sufficient Landau damping to suppress the instability. At the same time, we must avoid inducing a strong nonlinear resonance which would cause more particle loss. Hence, the rf PM tune $\nu_{m}$ is chosen to be the middle value of two neighboring resonance tunes $\nu_{s}$ and $3 \nu_{s}$, where $\nu_{s}$ is the synchrotron tune. $\nu_{m}$ is chosen to be $2 \nu_{s}$.

We start the PM at $10 \mathrm{~ms}$ after injection, and let it continue for $2 \mathrm{~ms}$. The amplitude of the rf PM is about 5 degrees with a modulation frequency of $10 \mathrm{kHz}$. With the rf phase modulation, the momentum spread of the bunch is effectively increased; this is evidenced with the successfully suppressed instability and simulation results of dynamics in longitudinal phase space, see Fig. 4. Two islands are formed and keep rotating in longitudinal phase space when rf PM is on; the islands' rotation causes the longitudinal bunch profile to change between single peaked and double peaked shape. The longitudinal bunch profile and its variation along time coincide with experimental observations. The relative momentum spread decreases as the energy of the proton bunch increases. Without rf PM, at some point around $10 \mathrm{~ms}$ after injection, the momentum spread is not big enough to suppress the instability; this is when the rf PM is applied.

\section{EXTRACTION OF TURN-BY-TURN BUNCH CENTROID DATA}

Both beam intensity and centroid position information can be extracted from the BPM signals. The summation of top and bottom BPM data is proportional to the intensity of the bunch, and the ratio of difference and summation is proportional to the deviation of the bunch's centroid from the BPM center. For dynamics analysis, we need to acquire the centroid position information on a turn-by-turn basis. Our BPMs are of split-shoe-box type. The length of the BPMs is $5 \mathrm{~cm}$. Since the length of the proton bunch is about 20 meters, much longer than that of the BPMs, the BPM signal is therefore proportional to the time derivative of the bunch intensity. Our analog-to-digital transform and data buffer system supports up to $1.25 \mathrm{GHz}$ sample rate for $3.2 \mathrm{~ms}$.

After integration of the raw BPM data, we should be able to calculate the centroid and intensity profile on a turnby-turn basis, but we found that there is a large-amplitude, low-frequency random noise, which shifts the baseline of the integrated BPM signal and makes the directly integrated BPM data unusable for comparing the centroid and intensity profiles between different turns. We need to further process the integrated BPM data before we use it: first, after integration, local minima and maxima are identified for intensity profile of each turn; then, with these minima and maxima points, boundaries of each turn can be obtained; finally compensations are made to remove the contributions from the baseline shifting for each turn. With these postprocessing steps complete, data of neighboring turns can be compared, but not of turns far away in time. This is because the measured data is naturally time based. Proton $\beta$ values increase from 0.3 to 0.7 over the acceleration cycle. Although the bunch length and particle spatial distribution typically do not change much over several hundred microseconds, bunch length shortens in time; therefore, we cannot overlap the intensity and centroid profiles to see the dynamics over thousands or even hundreds of turns. However, since we have separated beam data turn by turn, we can convert the time data of each turn to $\mathrm{rf}$ phase, that is from 0 to $2 \pi$ because the harmonic number is one. Then we can overlap data for many turns to see the centroid dynamics over a several millisecond range. 


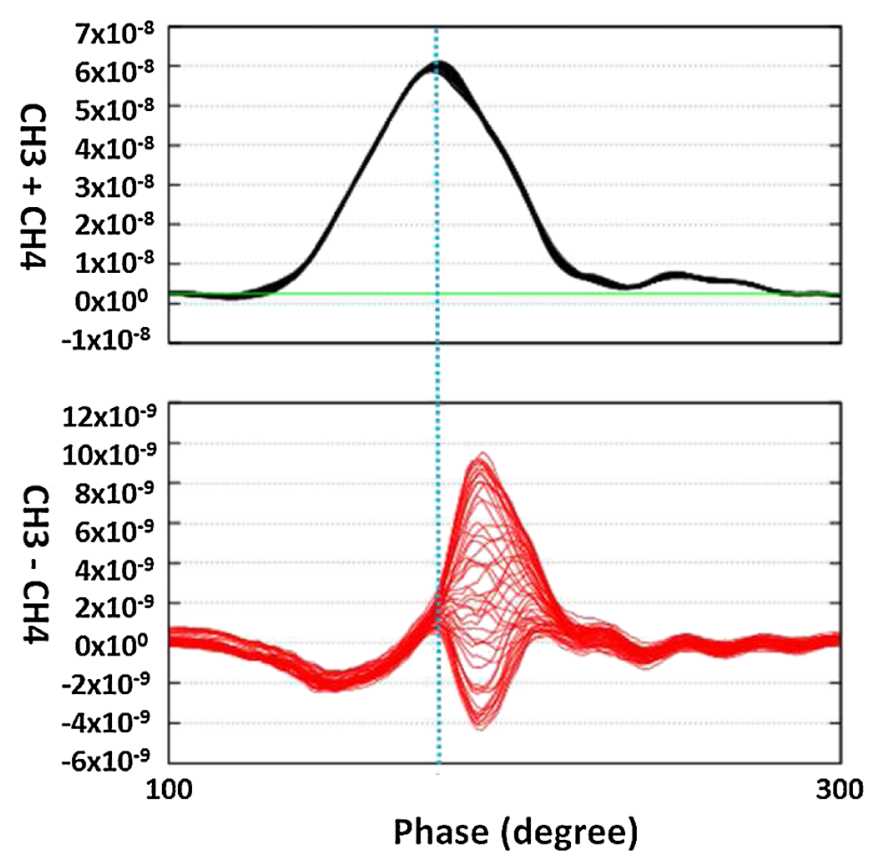

FIG. 5. (Color) The vertical centroid oscillations in the tail part, case 0 , measured at $13.88 \mathrm{~ms}$ for 50 consecutive turns. $\mathrm{CH} 3$ and $\mathrm{CH} 4$ are the top and bottom BPM signals.

\section{THE INSTABILITY OBSERVATION}

Now, we can look into the centroid dynamics with integrated and compensated BPM data.

When we turned off the rf PM, beam loss occurred; Figs. 5 and 6 show typical vertical bunch centroid dynamics observations with 50 consecutive turns data overlapped. At the same time, the horizontal centroid of the bunch is quite stable. Data in Figs. 5 and 6 are obtained from the same acceleration cycle.

The chromaticity $\xi$ was measured with the same ramping conditions when vertical bunch centroid oscillations occur with [5],

$$
\xi=\frac{d \nu}{d \delta}=\frac{d \nu}{\alpha_{c} \frac{d \Delta C}{C}}=\left(\eta+\frac{1}{\gamma^{2}}\right) R \frac{d \nu}{d R},
$$

where $\delta$ is the momentum deviation $d p / p, \eta$ is the phase slip factor, $\alpha_{c}$ is the momentum compaction factor, $C$ is the total path length, and $R$ is the RCS's average radius $(R=$ $6.83 \mathrm{~m})$. Both horizontal and vertical chromaticities at different moments during the ramp are measured. The horizontal chromaticities are negative or close to zero for the cycle. The vertical chromaticities when instability occurs are shown in Fig. 7.

In the bottom graphs of Figs. 5 and 6, it can be seen that 50 turns of data are enough to show the envelope of the oscillation of the bunch centroid. After computing the ratio of difference and summation of the BPM data, we found that the amplitude of the oscillations could be as large as one-fourth of the BPM vertical aperture. The vertical BPM size is $7 \mathrm{~cm}$. The beam's vertical emittance is measured at

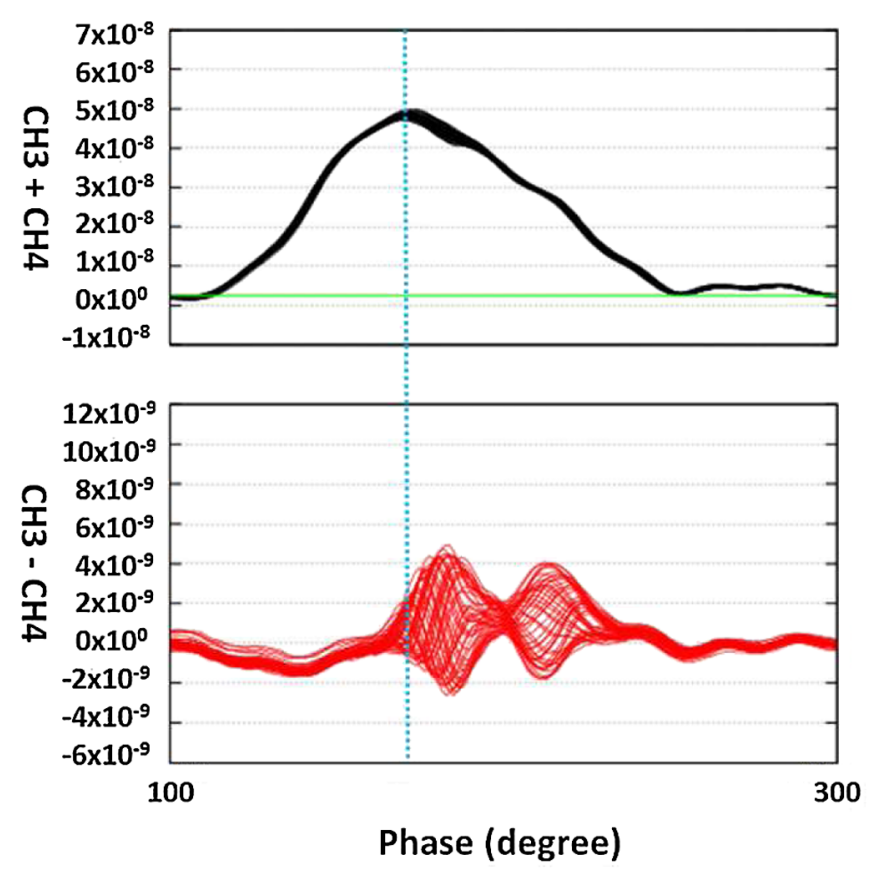

FIG. 6. (Color) The vertical centroid oscillations, case 1, measured at $13.72 \mathrm{~ms}$ for 50 consecutive turns. The blue line indicates that the front edge of the oscillation region locates at the intensity peak.

extraction to be approximately $60 \pi \mathrm{mm} \mathrm{mrad}$, which is close to the emittance of the beam 3-4 ms earlier in the acceleration cycle. Then the vertical beam size can be estimated to be $1.5 \mathrm{~cm}$ with $\beta_{y}=3.6 \mathrm{~m}$ calculated from a MAD model of the RCS. Hence, the transverse size of the proton bunch is relatively large; therefore, particles near the vertical edge of the beam can be lost.

The blue lines in Figs. 5 and 6 indicate that the front edge of the oscillation region is located at the intensity peak. The vertical centroid oscillation covers the tail part of

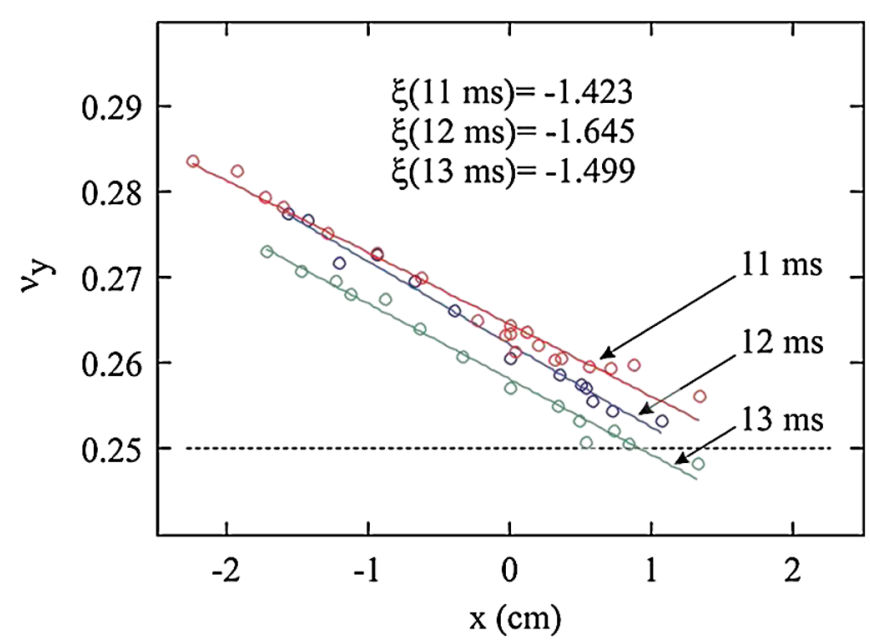

FIG. 7. (Color) Measured vertical chromaticities when instability occurs. $x$ is the radial deviation from the reference position. 


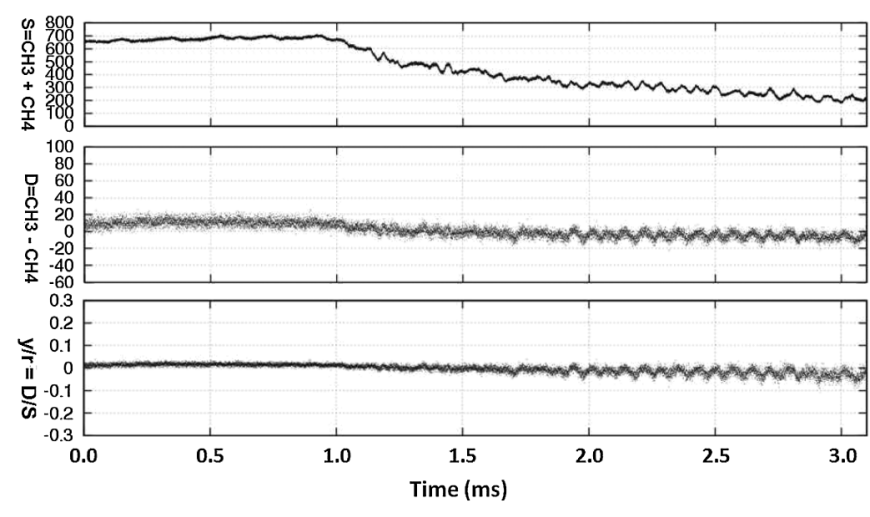

FIG. 8. Intensity and centroid of a slice with width of 5 degrees of rf phase at 152 degrees in the bunch head for $3.2 \mathrm{~ms}$ before extraction. Upper: sum BPM signal; middle: difference BPM signal; bottom: the ratio, centroid deviation. $\mathrm{CH} 3,4$ : the top and bottom BPM signals. In the figure, $t=0$ refers to approximately $11 \mathrm{~ms}$ in the cycle.

the bunch. One complete envelope period appears in Fig. 5, this type of oscillation is called case 0 . Because of the synchrotron motion, the bunch length and particle longitudinal distribution vary periodically. At a time $0.15 \mathrm{~ms}$ earlier, as shown in Fig. 6, the intensity peak is lower and the tail is longer. Two complete envelopes appear in Fig. 6; this type of oscillation is called case 1. Comparing the top graphs of Figs. 5 and 6, which are proportional to the intensity, we can see that the length of the tail part doubles when the bunch length is longer. Under the same experimental conditions, we have never observed centroid oscillations in the head part of the bunch.

More information can be obtained by analyzing just one slice at a time in the bunch. We choose the slice width to be 5 degrees of $\mathrm{rf}$ phase. BPM data of a chosen slice is obtained for a period of $3.2 \mathrm{~ms}$ just before extraction. Data of two slices are shown: Fig. 8 for one slice at 152 degrees in the middle of the bunch head and Fig. 9 for one slice at 212 degrees in the middle of the bunch tail.

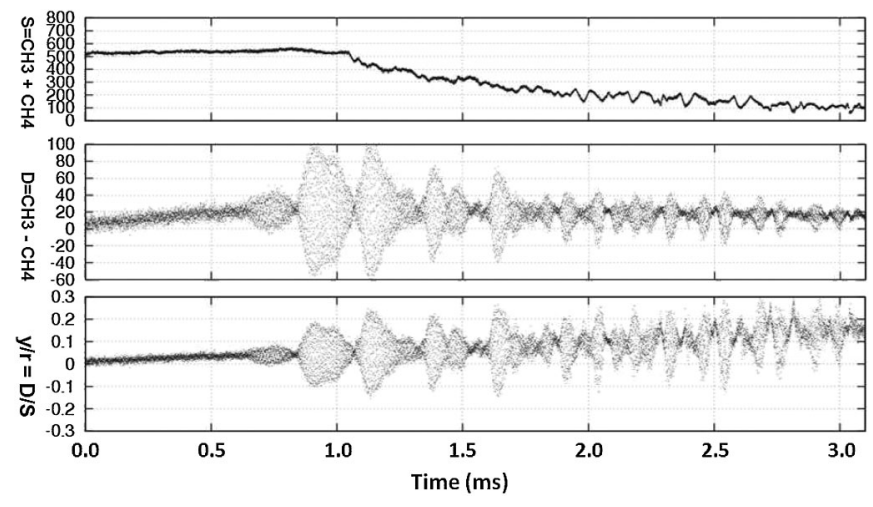

FIG. 9. Intensity and centroid of a slice with width of 5 degrees of rf phase at 212 degrees in the bunch tail for $3.2 \mathrm{~ms}$ before extraction. It can be seen that strong oscillations appear before bunch loss occurs. In the figure, $t=0$ refers to approximately $11 \mathrm{~ms}$ in the cycle.

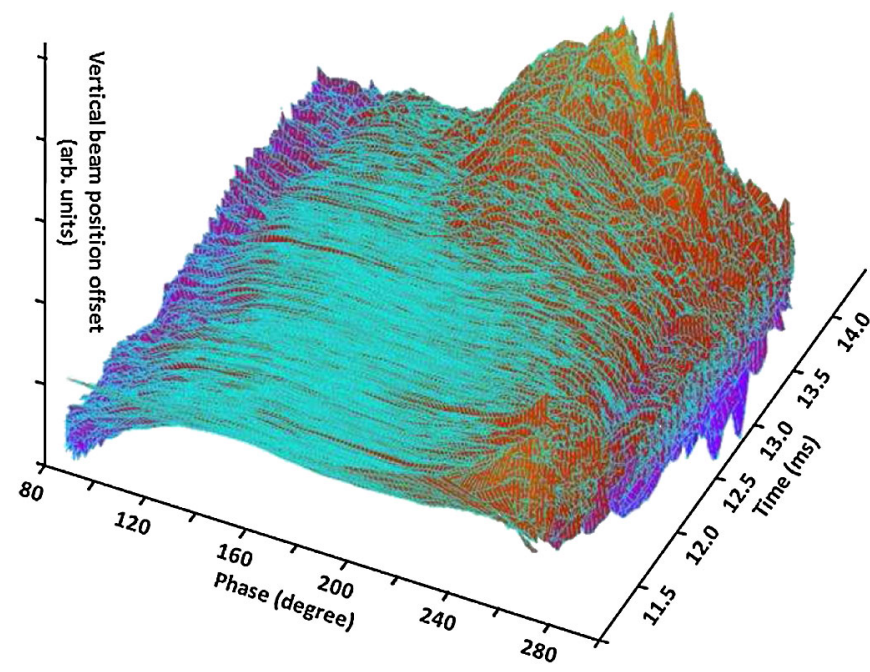

FIG. 10. (Color) The evolution of the vertical instability just before extraction. The phase is rf phase; it is used as longitudinal coordinate along the bunch, the head of the bunch has lower phase.

As it can be seen in Fig. 8, the centroid of the head slice is quite stable, though a small amplitude ripple appears in the centroid deviation. Figure 9 shows the strong oscillations in the tail slice. Soon after the oscillations begin, the bunch starts to lose particles in both the head and tail slices shown in the respective figures. Oscillations in the tail do not stop even when the bunch charge has dropped significantly. Both slices indicate a similar intensity reduction; however, a closer examination shows that the loss of particles in the tail slice precedes that in the head slice. This suggests that the exchange of particles between the head and the tail of the bunch by synchrotron motion caused the head intensity to droop, even though no instability occurred in the head. This is because when particles exchange between the head and the tail, the densities also exchange; hence, the head keeps giving more particles to the tail than those it receives from the tail, and the tail keeps losing particles.

Data for more slices in the bunch are obtained besides the above-mentioned two. When we look at neighboring slices (not shown in the figures), we notice that those closer to the tail of the bunch start oscillations and lose particles earlier. Eventually, the whole slice can be lost; in other words, the bunch is shortened as observed in Fig. 1. It is further noticed that in the middle and bottom graph of Fig. 9 the oscillations are modulated at a frequency close to the synchrotron frequency.

Although the synchrotron motion keeps damping the oscillation, the oscillations still develop from the tail to the center of the bunch over several synchrotron periods. The full evolution picture of the oscillation can be found in Fig. 10. The graph in Fig. 10 covers a period of $3.2 \mathrm{~ms}$ just before extraction. The average synchrotron frequency during this period is about $5 \mathrm{kHz}$; therefore, our observations cover approximately 16 synchrotron periods. 

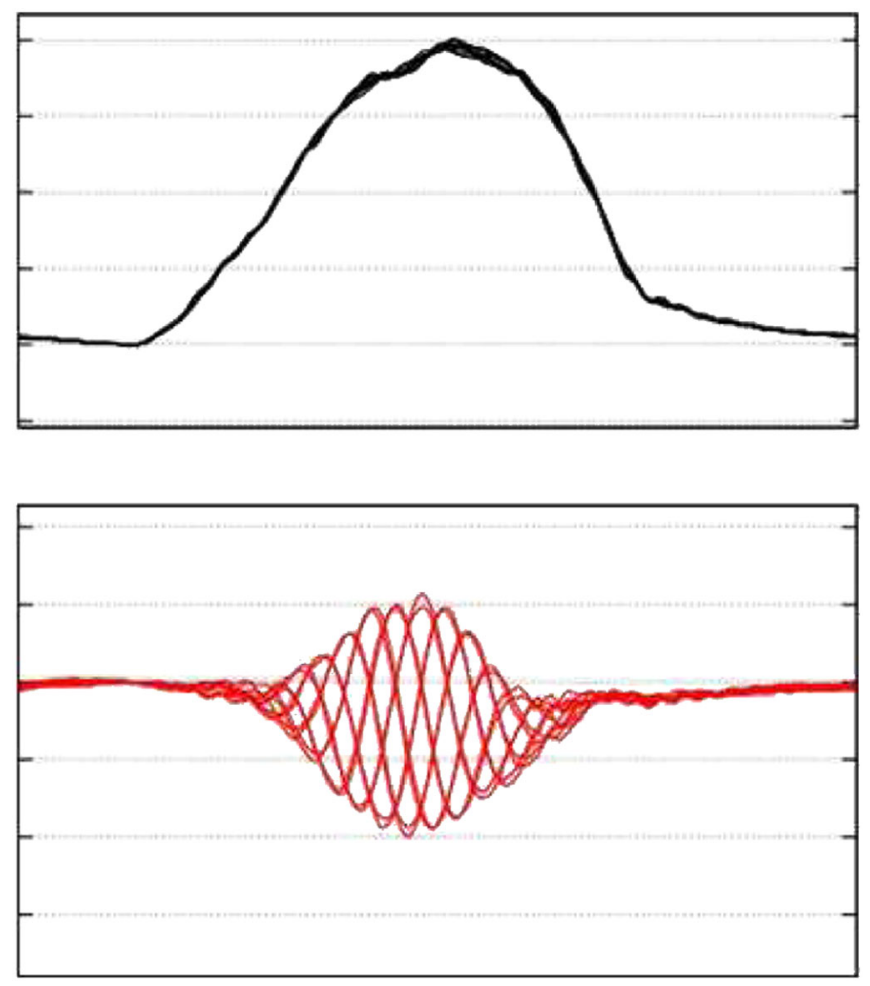

FIG. 11. (Color) The centroid oscillations in head-tail instability induced in the RCS ring. Upper: the sum of BPM signals; bottom: the difference of the BPM signals.

In the RCS, sextupoles were added to make the chromaticity negative, thereby suppressing the head-tail instability. In order to further distinguish the characteristics of the RCS instability, we turned off the sextupoles in the ring to purposely induce the head-tail instability. Using the same BPM data process method as described in the last section, we observed a classical head-tail instability, see Fig. 11. The bunch centroid oscillation in the induced head-tail instability covers the full bunch. This is distinct from the above-mentioned oscillation limited to the tail part of the bunch.

One possible explanation for the centroid oscillation is the electron cloud instability. The electron cloud and proton bunch are attracted to each other's potential well center. When the amplitude of the proton oscillation grows large enough, protons hit the beam pipe wall and are lost. Since the vertical size of the RCS beam pipe is smaller than the horizontal size, losses occur mainly in the vertical plane.

Before the head of the proton bunch returns to any given location in the RCS, some electrons may already be present in the volume of the vacuum pipe. Electrons can exist in the beam pipe for many reasons [6]. For example, electrons can be trapped on magnetic field lines in the combinedfunction, quadrupole, and sextupole magnets. In straight sections, electrons can be attracted to the positively charged, background gas ions remaining in the beam pipe. As the proton bunch approaches, low energy electrons are drawn toward the center of the proton potential.
As the proton bunch travels forward, the potential well gets deeper as the proton intensity increases. Those electrons inside the proton potential well oscillate within the well; they do not have enough energy to escape until the peak of proton bunch intensity passes. After the intensity peak, the potential well becomes shallower as the proton density decreases. Some of the captured electrons now have enough energy to escape from the proton bunch. Escaped electrons hit the beam pipe wall and produce secondary electrons. Once these secondary electrons come back and pass through the proton bunch, they will in turn produce additional secondary electrons. The number of electrons increases exponentially with this avalanche process [7]. At the same time, the depth of the electron induced potential well increases. Then collective interaction between proton bunch and electron cloud becomes significant especially if the electrons are bound by magnetic fields. Hence, the tail part of the proton bunch oscillates observably. The tail tip of the proton bunch sees more electrons than earlier slices, hence the tail tip always starts oscillation first. During a synchrotron period, when the tail portion of the bunch becomes long enough, more centroid oscillations can occur, then we observed case 1 , and it appears with case 0 alternately. After the whole proton bunch passes, most electrons are expelled outward by their own electric field, hit the beam pipe wall, and get lost before the arrival of the proton bunch head in the subsequent turn. Hence, the head of proton bunch has an almost clean start and is always stable as observed in the RCS. However, regarding measurements with the retarding field analyzer [8] at IPNS, we did observed what appeared to be electron signals, but we could not affect them with retarding potential up to $600 \mathrm{~V}$. We found no quantitative way to prove or disprove the possible existence of an electron cloud instability.

\section{SUMMARY}

We have presented an analysis of the vertical instability that limits the beam current in the IPNS RCS. Two cases of oscillations are observed. Both oscillations are confined in the tail part of the bunch, which is quite different from a classical head-tail instability. A possible explanation is a collective interaction between the proton bunch and electron cloud. Further experiments on identification of the existence of electron cloud in the instability process are needed.

\section{ACKNOWLEDGMENTS}

The authors thank members of the IPNS Accelerator group for fruitful comments and discussions.

[1] J. Gareyte and F. Sacherer, in Head-tail Type Instabilities in the CERN PS and Booster, 9th Conference on High Energy Particle Accelerators, Stanford, 1974. 
[2] C. Potts, IEEE Trans. Nucl. Sci. 32, 3107 (1985).

[3] A. Chao, Physics of Collective Beam Instabilities in High Energy Accelerators (John Wiley \& Sons, New York, 1993).

[4] J. Dooling, F. Brumwell, G. McMichael, and S. Wang, in Proceedings of the 21st Particle Accelerator Conference, Knoxville, 2005 (IEEE, Piscataway, NJ, 2005), p. 3375.

[5] S. Lee, Accelerator Physics (World Scientific, Singapore, 2004), 2nd ed.

[6] D. Neuffer, E. Colton, D. Fitzgerald, T. Hardek, R. Hutson, R. Macek, M. Plum, H. Thiessen, and T. S.
Wang, Nucl. Instrum. Methods Phys. Res., Sect. A 321, 1 (1992).

[7] V. Danilov, A. Aleksandrov, J. Galambos, D. Jeon, J. Holmes, and D. Olsen, in Workshop on Instabilities of High Intensity Hadron Beams in Rings, BNL, 1999, edited by T. Roser and S. Y. Zhang, AIP Conf. Proc. No. 496 (AIP, New York, 1999).

[8] K. Harkay and R. Rosenberg, Phys. Rev. ST Accel. Beams 6, 034402 (2003). 